\title{
Influencia de la composición mineralógica de puzolanas naturales en las propiedades de los cementos con adiciones
}

\section{The influence of natural pozzolana mineralogical composition in the properties of blended cement}

\author{
M. GENER RIZO, J. M. ALONSO LAVERNIA \\ Centro Técnico para el Desarrollo de los Materiales de Construcción y de la Empresa Constructora del Poder \\ Popular de Ciudad de La Habana, Cuba
}

Fecha de recepción: $3-\mathrm{V}-01$

Fecha de aceptación: 5-VII-01

CUBA

\section{RESUMEN}

La actividad puzolánica es la propiedad fundamental de las adiciones activas, pero para la selección de la misma se debe considerar, entre otros factores, su composición mineralógica, que influye no sólo en los constituyentes activos, sino también en muchas propiedades de los cementos.

En el presente trabajo, como material puzolánico se estudiaron 4 puzolanas naturales cubanas, las cuales fueron caracterizadas mediante difracción de Rayos $X$, análisis térmico y análisis químico; se evaluó, además, la actividad puzolánica mediante un método químico y otro fisicomecánico. Se prepararon cementos con diferentes contenidos de cada una de las puzolanas y se analizaron sus propiedades quimicas y fisico-mecánicas.

Se concluye que la composición mineralógica de las puzolanas influye de forma determinante en la actividad puzolánica y en las propiedades de los cementos mezclados; que los mejores desarrollos de resistencias en el tiempo y los menores requerimientos de agua en las mezclas, se obtienen cuando en las adiciones predomina la fase vitrea.

\section{SUMMARY}

The pozzolana activity is the main property of the active additions but, in order to select them, we have to consider between other factors-its mineralogical composition with a great influence, not only in the active component, but also in other cement properties.

In the present work we have studied 4 different Cuban natural pozzolanes, characterized with the help of X Ray diffraction and with thermic and chemical analysis. The pozzolanic activity was also evaluated through a chemical and physicomechanic method. Some cements were prepared with different contents of each one of the pozzolanics, and analysed their physicomechanic and chemical properties.

Finally, we found that the pozzolanics mineralogical composition has a great influence in the pozzolanic activity and in the properties of mixed cements. Also we found that it's possible to obtain the best resistances in the time and the smaller needs of water when the vitreous phase prevail in the additions.
PALABRAS CLAVE: cemento, puzolana, actividad, composición, resistencia

\section{INTRODUCCIÓN}

La producción de cementos con adiciones es ya una práctica común en la Industria del Cemento a nivel mundial. Las razones que justifican la utilización de adiciones son
KEYWORDS: cement, pozzolana, activity, composition, strength

tanto económicas (ahorro energético que se origina por la sustitución de una determinada cantidad de clínker por adiciones), como ecológicas (menor emisión de $\mathrm{CO}_{2}$ a la atmósfera), como técnicas (mejor comportamiento respecto a durabilidad e incluso mecánicamente resistente, 
en determinadas ocasiones, de los cementos con adiciones respecto a cementos exentos de ellas).

Dentro de los cementos con adiciones activas, el más antiguo que se conoce es el cemento puzolánico, que se remonta a la civilización romana, con el descubrimiento de las cenizas volcánicas de la región de «Puzzuoli» en Nápoles. De ahí el término de puzolana que se emplea en la actualidad para denominar a todos los materiales que tienen capacidad para reaccionar con la cal en presencia de agua y a temperatura ambiente para formar productos con propiedades hidráulicas (1) (2).

Con el incremento progresivo de la producción y empleo de los cementos con adiciones puzolánicas, y teniendo en cuenta la diversidad de estas adiciones, se ha hecho necesario intensificar las investigaciones relacionadas con los métodos de ensayo para evaluar su calidad junto con el conocimiento de sus propiedades (3-6).

La actividad puzolánica es la propiedad fundamental de estos materiales, pero para la selección de una puzolana se debe considerar, entre otros factores, su composición mineralógica, que influye no sólo en los constituyentes activos, sino también en la avidez por el agua, lo cual se relaciona directamente con la resistencia de morteros y hormigones, su laborabilidad, fisuración por secado y otras propiedades.

En el presente trabajo se estudia la influencia de la composición mineralógica de 4 tobas cubanas en las propiedades de los cementos con adiciones

\section{DESARROLLO DEL TRABAJO}

\subsection{Materiales empleados}

La localización en casi todo nuestro territorio de yacimientos de tobas, fue lo que impulsó a realizar su evaluación como adición activa en la producción de cemento.

Estos materiales son rocas vitroclásticas, de naturaleza sílico-aluminosa, constituídas en general por fragmentos de vidrio volcánico, alterados en mayor o menor grado a causa de los agentes atmosféricos. En el proceso de alteración del vidrio, se obtienen una serie de minerales nuevos: arcillosos (montmorillonita), zeolita, plagioclasas y otros. Son rocas porosas, de gran superficie específica y, en general, de color verde amarillento, con matices carmelitosos (7).

Se estudiaron 4 puzolanas de diferentes yacimientos: 1 de la región occidental, 2 de la central y 1 de la región oriental: «Los Congos», «Las Carolinas», «Tasajera», y «San Benito», respectivamente.

\subsection{Métodos de estudio}

La composición mineralógica se realizó mediante la difracción de Rayos X y Análisis Térmico. Los componentes fundamentales de las puzolanas de determinaron a través de análisis químico.

\subsubsection{Actividad puzolánica}

Para estudiar la actividad puzolánica de los materiales se analizó su fracción soluble a través de la determinación del contenido de óxidos solubles o activos después de un tratamiento ácido-base (4). La propiedad puzolánica se asocia con la magnitud de la parte soluble, constituída fundamentalmente por los componentes de la fase vítrea de las tobas $\left(\mathrm{SiO}_{2}, \mathrm{Al}_{2} \mathrm{O}_{3}, \mathrm{Fe}_{2} \mathrm{O}_{3}\right)$.

Además se utilizó un método físico mecánico desarrollado en el IETCC (5), que se basa en la resistencia a la compresión obtenida en un mortero preparado cal y puzolana, sometido a un ambiente de vapor de agua durante 72 horas.

\subsubsection{Cementos}

Se prepararon cementos en el laboratorio con adiciones de cada puzolana de 10,15 y $20 \%$ en peso, además de un cemento patrón sin adición, se empleó un clínker con un contenido de $\mathrm{C}_{3} \mathrm{~S}$ de $65 \%$ y yeso en una cantidad correspondiente al $2 \%$ de $\mathrm{SO}_{3}$.

Los cementos fueron elaborados en un molino de laboratorio hasta obtener una Superficie Específica según Blaine entre $3.200-3.600 \mathrm{~cm}^{2} / \mathrm{g}$ y un retenido en el tamiz de 90 micras menor del 5\%. Previamente el clínker fue molido a un tamaño menor de $0,5 \mathrm{~mm}$ y la toba y el yeso a un tamaño menor de 2-4 mm.

Se estudiaron las propiedades químicas, físicas y físicomecánica de los cementos. Los morteros fueron preparados según la ISO- RILEM, con una relación agua cemento constante e igual a 0.5 . Para analizar la influencia de la composición mineralógica sobre la trabajabilidad de las mezclas se midió la fluidez de cada uno de los morteros de cemento.

\section{RESULTADOS OBTENIDOS}

\subsection{Composición mineralógica y actividad puzolánica de las tobas}

En general, las tobas estudiadas están constituidas por una estructura zeolitíca del tipo clinoptilolita-heulandita $\left(8,98 \mathrm{~A}^{\circ}, 3,97 \mathrm{~A}^{\circ} ; 9,00 \mathrm{~A}^{\circ},, .92 \mathrm{~A}^{\circ}\right.$ reflexiones fundamentales) que varía en las diferentes muestras, además 
de plagioclasas $\left(3,18 \mathrm{~A}^{\circ}\right)$, montmorillonita $\left(11,2 \mathrm{~A}^{\circ}\right.$, $\left.3,47 \mathrm{~A}^{\circ}\right)$, cuarzo $\left(3,24 \mathrm{~A}^{\circ}, 4,27 \mathrm{~A}^{\circ}\right)$, calcita $\left(3, .03 \mathrm{~A}^{\circ}\right)$ y una fase vítrea dada por la banda difusa entre los $20^{\circ}-32^{\circ}$. En la toba de «San Benito» se determinó que estaba constituida fundamentalmente por una fase vítrea, lo que se deduce del nivel de fondo observado en el difragtograma, así como las escasas reflexiones correspondientes a las fases cristalinas.

Mediante el análisis térmico, se identificó un efecto endotérmico fuerte (curva ATD) con extremo en los $160^{\circ} \mathrm{C}-180^{\circ} \mathrm{C}$, asociado a la deshidratación de la estructura zeolítica y a la pérdida del agua interlaminar de la montmorillonita presente en las muestras; en el intervalo $480^{\circ} \mathrm{C}-620^{\circ} \mathrm{C}$, con extremo en $530^{\circ} \mathrm{C}$, un efecto endotérmico debido a la pérdida del agua hidroxílica de la montmorillonita. El efecto endotérmico en $780^{\circ} \mathrm{C}-800^{\circ} \mathrm{C}$ se identifica con la disociación de la calcita, el mismo está ausente en la muestra de toba de San Benito.

La estimación del contenido de zeolita se realizó a partir del efecto endotérmico en los $160-180^{\circ} \mathrm{C}$ y la correspondiente perdida de peso (curva TG) que lo acompaña. Se tuvo en cuenta que en este efecto se produce también la pérdida del agua interlaminar de la montmorillonita y que según la literatura (8) (9) en este intervalo de temperatura la montmorillonita pierde entre un 3-6\% . El cálculo se realizó teniendo en cuenta el valor máximo del $6 \%$, que restado a la perdida de peso total del efecto completo, permitió estimar el contenido de zeolita en las muestras estudiadas
También se identificaron 3 reacciones en el intervalo de los $200-400{ }^{\circ} \mathrm{C}$ que confirman la presencia de geles de sílice, aluminio y hierro presentes en la fase vítrea (10), las mismas estan acompañadas de una perdida de peso(curva TG). La mayor pérdida correspondió a la tơba de «San Benito» (22\%) y la menor a la de "Tasajera» $(10 \%)$, en la toba de «Los Congos» esas reacciones no se confirmaron(11) (12) (13).

En la Tabla 1, se muestra un resumen de la composición química y mineralógica de las muestras estudiadas.

La literatura (1) (4) (6) considera que las fases principales que intervienen en la acción puzolánica, son la fase vítrea y la estructura zeolítica, aunque esta última se manifiesta más lentamente; estas fases son consideradas activas. Las estructuras arcillosas como la montmorillonita son clasificadas como fases no activas e indeseables ya que incrementan los requerimientos de agua de las mezclas, disminuyen las fuerzas mecánicas e intercambian la cal de forma semejante a las zeolitas, pero sin formar productos de hidratación. Los minerales bien cristalizados como el cuarzo, la calcita y otros se consideran fases inertes.

En la Tabla 2 se muestran los resultados de la actividad puzolánica, la cual está íntimamente relacionada con la composición mineralógica de las muestras estudiadas.

Las tobas de «San Benito» y «Las Carolinas» clasificaron como muy activas, "Tasajera» como activa y «Los Congos» con actividad media. Según el ensayo físico

TABLA

Resumen de la composición química y mineralógica

\begin{tabular}{|l|l|l|l|l|}
\hline FASE & LOS CONGOS & CAROLINA & TASAJERA & SAN BENITO \\
\hline Vidrio & poco & abundante & poco & abundante \\
\hline Zeolita & moderada (15\%) & moderada (27\%) & abundante (57\%) & - \\
\hline Cuarzo & - & - & poco & escaso \\
\hline Montmonllonita & abundante & Poca & poca & poca \\
\hline plagioclasa & moderada & Poca & poca & poca \\
\hline calcita & moderada (12\%) & poca (5\%) & escasa (2\%) & - \\
\hline $\mathrm{SiO}_{2}$ & $42,46 \%$ & $65,03 \%$ & $65,64 \%$ & $73,43 \%$ \\
\hline $\mathrm{SiO}_{2},+\mathrm{Al}_{2} \mathrm{O}_{3},+\mathrm{Fe}_{2} \mathrm{O}_{3}$ & $59,68 \%$ & $80,27 \%$ & $79,61 \%$ & $83,25 \%$ \\
\hline
\end{tabular}

TABLA 2

Actividad puzolánica




mecánico, la mayor actividad puzolánica correspondió a la toba de «San Benito» donde predomina el vidrio volcánico, le sigue en orden decreciente «Las Carolinas» que posee abundante vidrio y moderada zeolita, después «Tasajera «con abundante zeolita y poco vidrio; la menor actividad la tuvo la toba «Los Congos» que posee poco vidrio y moderada zeolita.

\subsection{Propiedades de los cementos}

A continuación, en las Tablas 3 y 4 se resumen algunas de las propiedades químicas físicas y físico mecánicas de los cementos estudiados.

Se observa un incremento en los valores de los residuos insolubles y las pérdidas por ignición en la medida que se incrementa la adición de puzolana, asociado a la propia composición mineralógica de estos materiales. Este aumento es más significativo en aquellas muestras que contienen cantidades considerables de montmorillonita y zeolita.

Con relación a la consistencia normal, también se incrementa con la adición de puzolana; las zeolitas manifiestan gran demanda de agua debido a su estructura porosa y abierta, así como su alta superfiecie específica que permite la quimisorción de agua en las partículas más finas. A diferencia de la montmorillonita, que también manifiesta una alta exigencia de agua, interviene en la actividad puzolánica. A igual contenido de adición de puzolana, la mayor consistencia normal correspondió a los cementos elaborados con la puzolana de Los Congos, lo que indica que la misma necesita mayor cantidad de agua para lograr una pasta de consistencia normal

El peso específico disminuye con la adición de puzolana, factor que debe tenerse en cuenta en los cálculos de las dosificaciones de hormigones; los tiempos de fraguado inicial y final, se retardan con el incremento de la adición.

Es bien conocido que las adiciones decrecen la trabajabilidad de las mezclas, en mayor grado cuando éstas poseen constituyentes con más necesidad de agua, como es el caso de las zeolitas y montmorillonita.

En las primera edades de endurecimiento, los valores obtenidos de resistencia decrecen con la adición de puzolana; con adiciones del 10\% esta disminución es poco significativa en los cementos que contienen la toba de «San Benito» y «Las Carolinas» que poseen mayor contenido de fase vítrea, a partir de los 7 días, los valores de resistencias son prácticamente iguales.

En el caso de la toba de «Tasajera» a partir de los 90 días los valores de resistencia con el $10 \%$ de puzolana se iguala al Portland puro. Con la de "Los Congos", se obtuvieron los menores valores de resistencia; esta muestra presentó

TABLA 3

Propiedades químicas y físicas de los cementos

\begin{tabular}{|c|c|c|c|c|c|c|}
\hline \multirow{2}{*}{$\begin{array}{l}\text { TIPO DE } \\
\text { CEMENTO }\end{array}$} & \multicolumn{2}{|c|}{$\begin{array}{l}\text { PROPIEDADES } \\
\text { QUIMICAS }\end{array}$} & \multicolumn{4}{|c|}{ PROPIEDADES FÍSICAS } \\
\hline & $\begin{array}{r}\text { R.I } \\
\% \text { \% p }\end{array}$ & P.P.I & $\begin{array}{l}\text { P.E } \\
\text { g/cm }\end{array}$ & $\begin{array}{l}\mathrm{CN} \\
\%\end{array}$ & $\underset{(\min )}{\text { TFI }}$ & $\begin{array}{l}\text { TFF } \\
\text { (h) }\end{array}$ \\
\hline Portland Puro & 0.93 & 0.85 & 3.15 & 23 & 90 & $2 \mathrm{~h} 30^{\prime}$ \\
\hline $10 \%$ Los Congos & 4.92 & 2.87 & 3.12 & 27 & 120 & $3 \mathrm{~h} 30^{\prime}$ \\
\hline $15 \%$ Los Congos & 7.19 & 5.82 & 3.08 & 29 & 135 & $4{\mathrm{~h} 15^{\prime}}^{\prime}$ \\
\hline $20 \%$ Los Congos & 10.63 & 7.74 & 2.98 & 32 & 145 & $4 h^{4} 40^{\prime}$ \\
\hline 10\% Carolina & 2.81 & 1.98 & 3.13 & 25 & 110 & $3 \mathrm{~h}$ \\
\hline $15 \%$ Carolina & 4.05 & 3.18 & 3.09 & 27 & 116 & $3 \mathrm{~h} \mathrm{45}$ \\
\hline $20 \%$ Carolina & 6.15 & 5.84 & 3.03 & 28 & 132 & $4{\mathrm{~h} 15^{\prime}}^{\prime}$ \\
\hline $10 \%$ Tasajera & 3.28 & 2.15 & 3.13 & 27 & 112 & $3 \mathrm{~h} 30^{\prime}$ \\
\hline $15 \%$ Tasajera & 5.36 & 3.96 & 3.10 & 28 & 125 & $4 h$ \\
\hline $20 \%$ Tasajera & 7.61 & 6.04 & 3.06 & 30 & 142 & $4{\mathrm{~h} 35^{\prime}}^{\prime}$ \\
\hline $10 \%$ San Benito & 1.45 & 1.23 & 3.10 & 24 & 100 & $3 \mathrm{~h}$ \\
\hline $15 \%$ San Benito & 2.87 & 2.89 & 3.05 & 26 & 110 & $3 \mathrm{~h} 40^{\prime}$ \\
\hline $20 \%$ San Benito & 5.66 & 4.38 & 2.95 & 27 & 130 & $4 \mathrm{~h} 5^{\prime}$ \\
\hline
\end{tabular}

Nota:

R.I. Residuo insoluble

P.P.I Perdida por ignición

P.E. Peso específico

CN Consistencia normal

TFI y TFF Tiempo de fraguado inicial y final 
TABLA 4

Resistencia a la compresión en morteros de cemento. Relación agua/cemento $=0,5$

\begin{tabular}{|c|c|c|c|c|c|c|}
\hline TIPO DE & FLUIDEZ & \multicolumn{5}{|c|}{ RESISTENCIA A COMPRESIÓN MPa } \\
\hline \multirow[b]{2}{*}{ CEMENTO } & \multirow[b]{2}{*}{$(\%)$} & & & 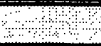 & + & प्रि" \\
\hline & & $24 \mathrm{~h}$ & $3 \mathrm{~d}$ & $7 \mathrm{~d}$ & $28 d$ & $90 \mathrm{~d}$ \\
\hline Portland Puro & 153 & 15.3 & 26.1 & 33.5 & 44.2 & 50.3 \\
\hline $10 \%$ Los Congos & 141 & 8.7 & 17.9 & 35.8 & 37.3 & 46.5 \\
\hline $15 \%$ Los Congos & 133 & 7.6 & 14.3 & 23.1 & 34.6 & 40.6 \\
\hline $20 \%$ Los Congos & seco & 5.4 & 12.1 & 18.4 & 30.6 & 37.3 \\
\hline $10 \%$ Carolina & 148 & 13.8 & 24.8 & 32.7 & 43.4 & 51.4 \\
\hline $15 \%$ Carolina & 143 & 11.7 & 21.6 & 29.4 & 41.8 & 49.8 \\
\hline $20 \%$ Carolina & 135 & 8.9 & 19.3 & 27.3 & 40.5 & 48.3 \\
\hline $10 \%$ Tasajera & 145 & 11.4 & 20.5 & 29.9 & 39.6 & 49.9 \\
\hline $15 \%$ Tasajera & 137 & 9.3 & 18.4 & 26.5 & 37.1 & 45.8 \\
\hline $20 \%$ Tasajera & 133 & 6.5 & 16.7 & 24.6 & 35.2 & 42.4 \\
\hline $10 \%$ San Benito & 150 & 14.1 & 25.7 & 32.9 & 44.1 & 52.8 \\
\hline $15 \%$ San Benito & 146 & 12.9 & 24.3 & 31.6 & 42.8 & 51.6 \\
\hline $20 \%$ San Benito & 140 & 10.6 & 21.7 & 28.3 & 41.4 & 50.4 \\
\hline
\end{tabular}

la menor actividad puzolánica y los mayores contenidos de montmorillonita

\section{CONCLUSIONES}

El conocimiento profundo y detallado de las propiedades de las puzolanas permitirá su selección para la producción de cementos con adiciones y la obtención de los mejores resultados en los hormigones. Así, en las puzolanas Los Congos, Carolina, Tasajera y San Benito se confirma que su composición mineralógica influye de forma determinante en su actividad puzolánica y en las propiedades de los cementos que las contienen. Los re- sultados obtenidos en el presente trabajo permiten afirmar que:

Los mejores desarrollos de resistencia en el tiempo y los menores requerimientos de agua se obtuvieron en los cementos con adición de la puzolana de San Benito en la que predomina la fase vítrea, seguidos de los que contienen la de Carolina y, posteriormente, la de Tasajera.

La mayor presencia de montmorillonita en la puzolana Los Congos respecto al resto de las puzolanas estudiadas, incrementa la necesidad de agua del mortero e influye negativamente en los valores de resistencia.

\section{BIBLIOGRAFÍA}

(1) Massazza, F; Costa, U. Aspecto de la actividad puzolánica y propiedades de los cementos puzolánicos. Seminario sobre la química de los cementos, Wexham Springs, Reino Unido. Sept. 1977.

(2) Calleja, J; Apología de los conglomerantes puzolánicos. Revista Cemento Hormigón, no. 386. Mayo 1966.

(3) Sersale, R; Aiello, R. Natural zeolites as active additions to the Portland clinker. $8^{\circ}$. Congreso Internacional de la Química del Cemento. Volumen VI . Río de Janeiro, Brasil. 1986.

(4) Gener, M. Calidad de las tobas para cemento puzolánico. Informe PPE-046.12, CTDMC. La Habana, Cuba, Julio 1985.

(5) Luxan, M. P; Sánchez de Rojas, M.I.. Estudio de la aptitud de un material como adición activa al cemento. Revista Materiales de Construcción, Vol. 35. \#200. 1985.

(6) Rabilero, A. Las puzolanas. Editorial Oriente. La Habana, Cuba, 1987.

(7) Coutin, D; Brito A.; Los yacimientos de puzolana naturales de las Villas, Camagüey y Oriente. Serie Geológica \#24 ACC, IGP, La Habana, Cuba, 1976.

(8) Kuzniarova, A.L.; Análisis Térmico de minerales arcillosos y otros silicatos, Departamento de Geología, Universidad Politécnica de Varsovia, Polonia, 1967.

(9) Schultze, D; Análisis Térmico Diferencial. Universidad Politécnica de Varsovia, Polonia, 1974.

(10) Escalona, I: Composición de la fase vítrea de las tobas. III Ciclo de Conferencias Científicas del Instituto Superior Politécnico José Antonio Echevarria. La Habana, Cuba, 1982.

(11) Mackenzie, R.C. y Meldan, R.; Miner. Mag. 33, pág. 145-157, 1962.

(12) Schewertman, V; Nature London 22, pág. 645-646, 1966.

(13) Gener, M.; Composición mineralógica de puzolanas naturales cubanas. Boletín CIEC 3, La Habana, Cuba, 1979

$$
\text { *** }
$$

Nota: Parte de este trabajo fue realizado en el Instituto de Ciencias de la Construcción Eduardo Torroja durante la estancia de los autores en esa institución, bajo la asesoría y consultoría de los Doctores Tomás Vazquez Moreno, María Teresa Blanco Valera y María del Pilar Luxán. 